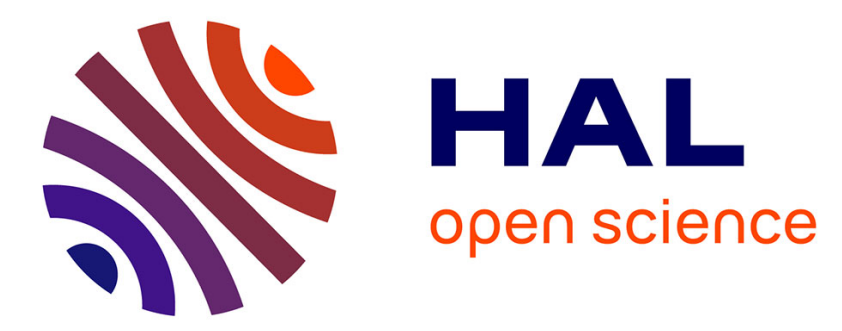

\title{
Porous Macroligands: Materials for Heterogeneous Molecular Catalysis
}

F. Wisser, Y. Mohr, J. Canivet, Elsje Alessandra Quadrelli

\section{To cite this version:}

F. Wisser, Y. Mohr, J. Canivet, Elsje Alessandra Quadrelli. Porous Macroligands: Materials for Heterogeneous Molecular Catalysis. ChemCatChem, 2020, 12 (5), pp.1270-1275. 10.1002/cctc.201902064 . hal-02447092

\section{HAL Id: hal-02447092 \\ https://hal.science/hal-02447092}

Submitted on 11 Jun 2020

HAL is a multi-disciplinary open access archive for the deposit and dissemination of scientific research documents, whether they are published or not. The documents may come from teaching and research institutions in France or abroad, or from public or private research centers.
L'archive ouverte pluridisciplinaire HAL, est destinée au dépôt et à la diffusion de documents scientifiques de niveau recherche, publiés ou non, émanant des établissements d'enseignement et de recherche français ou étrangers, des laboratoires publics ou privés. 


\title{
Porous Macroligands: Materials for Heterogeneous Molecular Catalysis
}

\author{
Florian M. Wisser, ${ }^{*[a]}$ Yorck Mohr, ${ }^{[a]}$ Elsje Alessandra Quadrelli ${ }^{[b]}$ and Jérôme Canivet ${ }^{\star[a]}$
}

\begin{abstract}
The integration of catalytically active centers into a solid support without any loss of performance compared to the homogeneous analogue remains a major challenge. In this context, solid macroligands can be regarded as the key element combine the advantages of homogeneous and heterogeneous catalysis. Porous materials such as periodic mesoporous organosilica, metal-organic frameworks, porous organic polymers or covalent-organic frameworks fulfill all requirements of a macroligand acting as a solid ligand in a molecular complex. They all can be tuned at the molecular scale to adapt the properties of the catalyst for a given application. This important feature also allows to directly compare different macroligands, regardless of their nature, and to choose the most appropriate for a target application.
\end{abstract}

\section{Homogeneous and heterogeneous catalysis}

In a historical retrospective, heterogeneous catalysts have been developed for applications in bulk chemical production, petrochemistry, and often under harsh conditions in terms of temperature and pressure. Until today zeolites as acidic catalysts remain unparalleled for the cracking of petrochemical fractions, and metal (nano-)particles supported on oxides as catalysts in reforming or hydrogenation reactions. It is their mechanical properties combined with an exceptional stability and activity that make heterogeneous catalysts prone for large industrial applications. Due to their shapeability (wires, nets, spheres, extrudates, etc.) they can be easily implemented into continuous flow processes and their thermal stability allows for a simple thermal regeneration. However, their ill-defined active sites often lack of versatility, thus limiting the adjustment of their activity and selectivity.

In contrast, classical homogeneous catalysts are commonly based on organic, and often sophisticated, N- or P-containing ligands, like functionalized phosphines or (bi)pyridines, coordinated to (noble) metal centers making their large scale production relatively costly. This comes with the advantage of controlling at the molecular level their activity, selectivity and even regio- and stereochemistry and ultimately with the high value

[a] Dr. F. M. Wisser, Mr. Y. Mohr, Dr. J. Canivet Université de Lyon, Université Claude Bernard Lyon 1, CNRS, IRCELYON - UMR 5256, 2 Avenue Albert Einstein, 69626 Villeurbanne, France

E-mail: florian.wisser@ircelyon.univ-lyon1.fr jerome.canivet@ircelyon.univ-lyon1.fr

[b] Dr. E.A. Quadrelli

Université de Lyon, Université Claude Bernard Lyon 1, CPE Lyon, CNRS, C2P2 - UMR 5265, 48 Bvd du 11 novmebre 1918, 69616 Villeurbanne, France added of the resulting products. Typically, homogeneous catalysts appear in liquid phase batch-type processes for fine chemicals. However, because of their homogeneous nature, their separation from the product and their recycling are difficult to achieve.

To combine the advantages of both types of catalysis, one has to master the design of robust materials, i.e. mechanically, chemically and thermally stable, while at the same time applying the high synthetic control known from molecular chemistry. To address this challenge, a key relies on the heterogenization of well-defined molecular catalysts keeping their activity and selectivity at least equal to those of homogeneous analogues.

\section{A porous solid as ligand}

In the nineties, the surface organometallic chemistry (SOMC) opened a new area in heterogeneous catalysis. SOMC has been introduced by Basset and co-workers as the reactivity of an organometallic (or coordination) compound with chemical groups at the surface of oxides, sulfides or zeolites (Figure 1a) ${ }^{[1]}$ With SOMC, the elementary steps of heterogenized catalysis would obey the rules of molecular chemistry with however the restrictions that the oxide support is an additional metal-oxo rigid ligand and that free surface species can interact with grafted catalytically active sites. ${ }^{[2-4]}$

To prevent eventual effect of the interactions of the active site with the oxide surface, the most drastic strategy is to get rid of the support itself. To generate self-supported molecular catalysts, the ligand of the target molecular complex is used as a building unit to construct a molecularly defined host matrix whose porosity ensure site accessibility (Figure 1b).

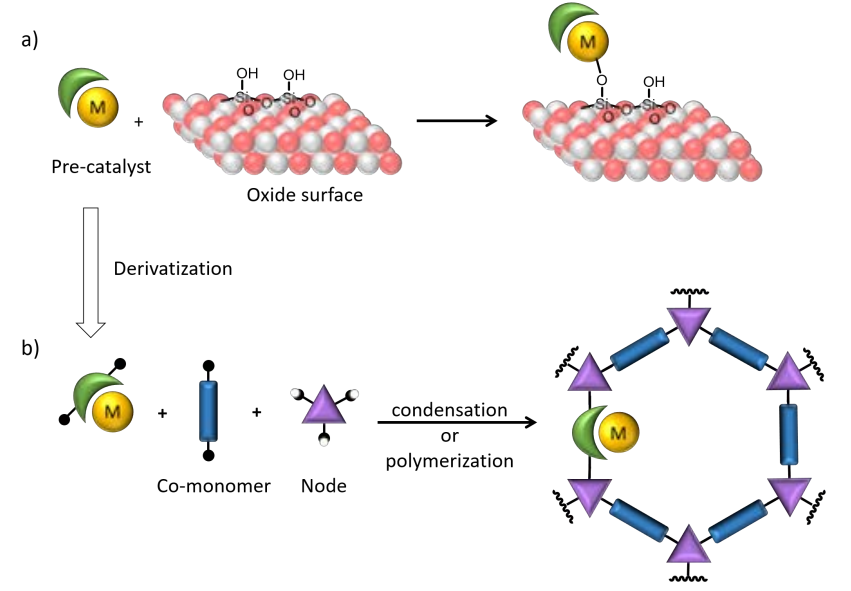

Figure 1. Surface organometallic chemistry (a) versus porous macroligands (b) for the heterogenization of molecular catalysts. 
In this regard the whole solid matrix becomes a so-called porous macroligand, a concept which is derived from polymer chemistry. ${ }^{[5,6]}$ Here, the catalyst retains its molecular character while being embedded within a porous framework.

At the interface between materials and molecules, porous macroligands gather key features from these two fields. Macroligand materials show a permanent porosity ensuring site accessibility throughout the solid network. Because of their high porosity, porous macroligands allow reaching tremendous site density, in catalytic sites per catalyst mass, since no support bulk volume remain unused, in contrast to classical oxide supports. Moreover, macroligands are built up by the repetition of molecular units, with some being the molecular complex, using condensation or polymerization reactions. Macroligands thus allow for a modular approach by the use of co-monomers resulting in single-site active species via spatial dilution and in the possibility to introduce side functionalities for a tailored, and ultimately predictable, reactivity. ${ }^{[7-10]}$

These important properties have so far rarely been considered, especially when comparing different host materials.

\section{Variety of porous macroligands}

To minimize interactions with oxide surface while embedding molecular ligand into the framework, the first strategy to design a porous macroligand is to reduce the oxide surface to $1 \mathrm{D}$ or $3 \mathrm{D}$ metal-oxo clusters linked by the organic ligands found in molecular complexes.

Inspired by traditional inorganic support such as mesoporous silica and zeolites, periodic mesoporous organosilica (PMO) and metal-organic frameworks (MOF) are hybrid organic-inorganic solids made of metal oxide/hydroxide building units bridged by organic linkers. PMO are siliceous materials prepared by polycondensation of a bis-silane organic molecules in the presence of a template to form one dimensional channels where the organic linker is embedded into the silica pore walls. ${ }^{[11]}$ In contrast, MOF materials are prepared by self-assembly between metal ions or metal oxo-clusters as nodes and suitable polytopic organic molecules, often of rigid nature, as linker moieties to form one-dimensional channels or three-dimensional cavities. As a result, the bonds between these nodes (also called secondary building units, SBU) and the linker moieties (linkers) are of coordinative nature (typically: $\mathrm{M}-\mathrm{N}$ or $\mathrm{M}-\mathrm{O}$ ). Since the making and breaking of coordinative bonds displays a dynamic equilibrium, the bond formation is reversible. ${ }^{[12]}$ These factors allow for the conception of specific porous network geometries on the nanometer scale. Exploiting both the rich field of coordination chemistry and the broad libraries of polytopic organic linkers gives rise to an almost infinite number of MOF structures.

The high versatility of PMO and MOF make them very appealing as macroligands for active site tuning in advanced catalytic applications. However, their constitutive metal-oxygen bonds can rapidly become their Achilles Heel when hydrolysis or competitive coordinating reactants come into play.

Overcoming such stability issue, covalent organic frameworks (COF) and porous organic polymers (POP) are purely organic porous solids built up entirely on covalent bonds (typically C-C, C$\mathrm{N}, \mathrm{C}-\mathrm{O}$ or $\mathrm{B}-\mathrm{O}$ ). The classical distinction between them lies in the way they are synthesized and hence in their resulting structural properties. ${ }^{[13,14]}$ Historically, COFs are built from partial reversible condensation reactions making them (semi)crystalline materials. ${ }^{[15]}$ In turn, the networks in POP stem from irreversible covalent bond formation reactions, e.g. carbon-carbon coupling reactions, yielding to amorphous solids with however well-defined building units at the molecular level. ${ }^{[16]}$ Finally, POP offer the possibility to introduce co-monomers with additional functionalities and geometries, paving the way for sophisticated fine-tuning of active sites in an enzyme-like fashion.

The application potential, stability and recyclability of single-site catalysts immobilized inside MOF, COF and POP have been recently reviewed. ${ }^{[16-19]}$ The rational comparison between these families of porous materials requires unifying them under the general concept of macroligands.

Regarding the design of macroligands, porous structures made from the repetition of moieties bearing a coordination motif like bipyridines, phenanthrolines or phosphines are of great interest since they are among the most widely used chelating ligands in organometallic chemistry. In particular, its synthetic availability, its substituent positional versatility and its chemical stability make 2,2'-bipyridines (Bpy) the building block of choice for further metal cation coordination. ${ }^{[20]}$

Figure 2 shows selected examples of the wide diversity of bpybased porous solids that can be envisioned as macroligands for coordination complexes. These porous macroligands can be classified in terms of compositions (hybrid organic-inorganic or organic) and in terms of pores connectivity (1D channels or 3D cavities). Among hybrid materials, BpyPMO ${ }^{[21-23]}$ or MOF-253 ${ }^{[24]}$ present 1D channels which can induce transport limitations to the active site in the case of bulky molecules when UiO-67-Bpy ${ }^{[25]}$ offers a 3D porosity. Similarly, purely organic compounds like $\mathrm{BpyCTF}^{[26]}$ (CTF: covalent triazine framework) and TpBpy ${ }^{[27]}$ made of $1 \mathrm{D}$ channels with additional functionalities at the node. In contrast, 3D polymers like POP-Bpy ${ }^{[28]}$ and BpyMP-1 ${ }^{[7]}$ offer a high accessibility to the active site. 

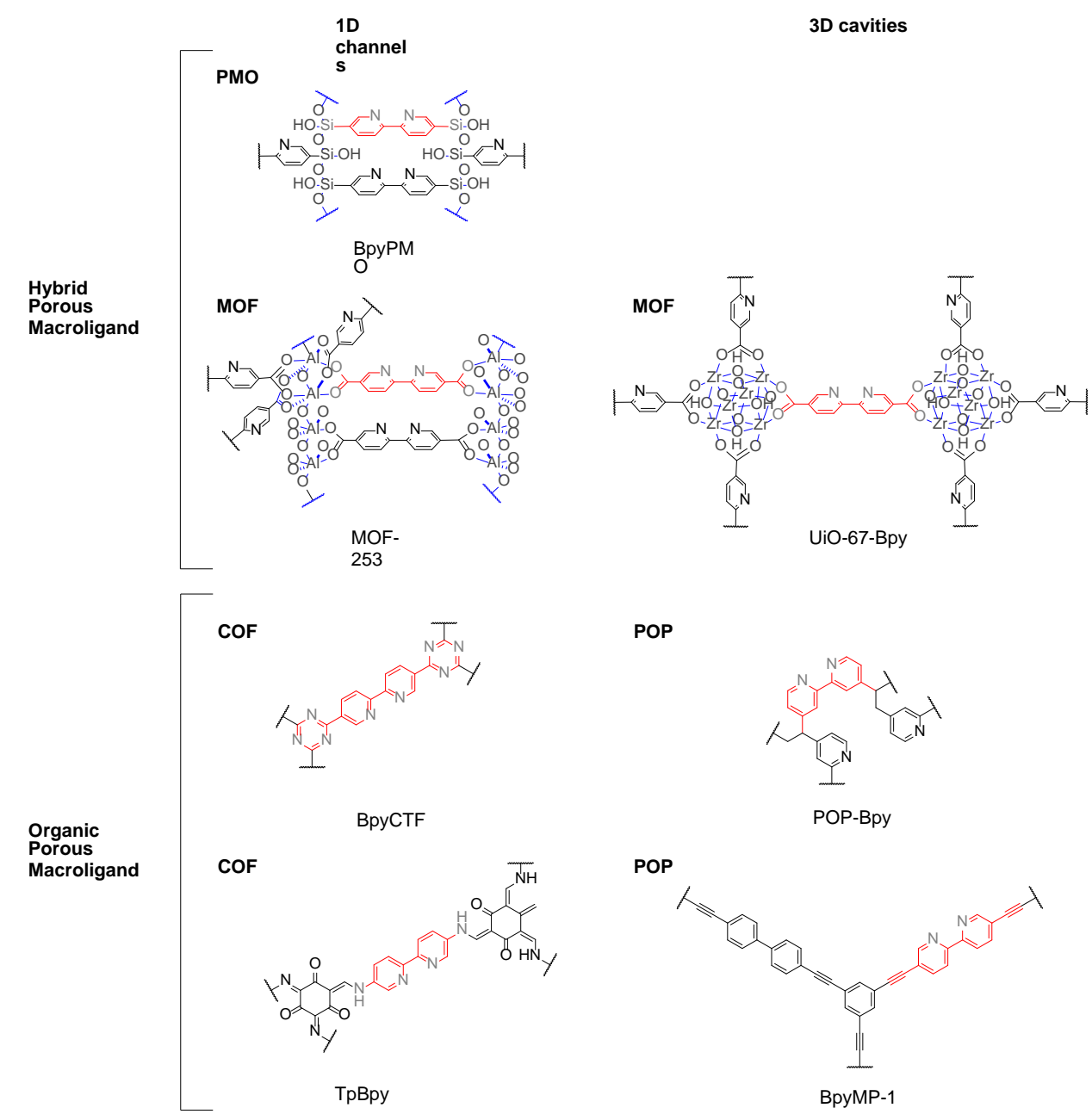

POP
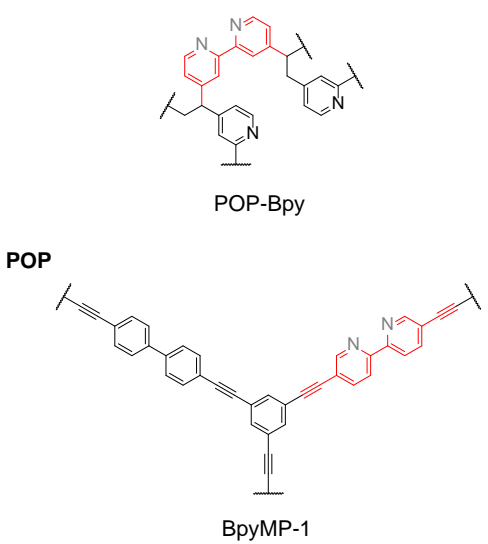

Figure 2. Repeating units in selected examples of bipyridine-based hybrid organic-inorganic and fully organic porous macroligands.

\section{Understanding heterogenized catalysts at the molecular level}

When comparing different porous macroligands at the molecular scale with respect to their influence on the catalytic activity, few additional requirements have to be taken into account: ${ }^{[7,29-31]}$

(i) The free diffusion inside the porous framework ensuring a thermodynamic control of the catalytic reaction;

(ii) A similar reactant uptake in the studied porous macroligands;

(iii) The interactions at the interface between adsorbate (solvent and reactants, liquid or gas) and the studied porous solids.

In two recent studies by our team, a series of heterogeneous catalysts based on Bpy macroligands was presented..$^{[7,8]}$ These catalysts where designed by constructing different types of environments around the Bpy-moieties resulting in MOF and
POP-based porous macroligands. As of MOF-macroligands UiO67(Zr) and MOF-253(Al) were prepared. Further, the POPs BpyMP-1 and BpyMP-2 were used. As mentioned above the more robust synthetic pathway of POPs allowed to introduce additional functional groups on the bipyridine moiety in BpyMP materials. All macroligands were then metallated with a $\mathrm{Rh}-\mathrm{Cp}^{*}$ precursor in order to obtain the metalorganic complex $\left[\mathrm{Rh}\left(\mathrm{Cp}^{\star}\right)(" \mathrm{Bpy}) \mathrm{Cl}\right]^{+}$in different versions.

For two different prototypic reactions (photocatalytic $\mathrm{CO}_{2}$ reduction and transfer hydrogenation reaction), the same trend of catalytic activity was observed: BpyMP >> UiO-67 > MOF-253. Even though the catalytic site, a Bpy-bound Rh-Cp* unit, seems to be the same in all cases, the electronic environment of the active sites differs significantly. The tool that was used to describe those changes in electron density is the Hammett parameter $\sigma$. The Hammett parameter $(\sigma)$ assigns to different substituents in different positions dimensionless numbers based on their electron-withdrawing or electron-donating properties. ${ }^{[32]}$ It therefore becomes possible to rationalize the influence of electron-withdrawing and electron-donating groups onto the catalyst's performance. 

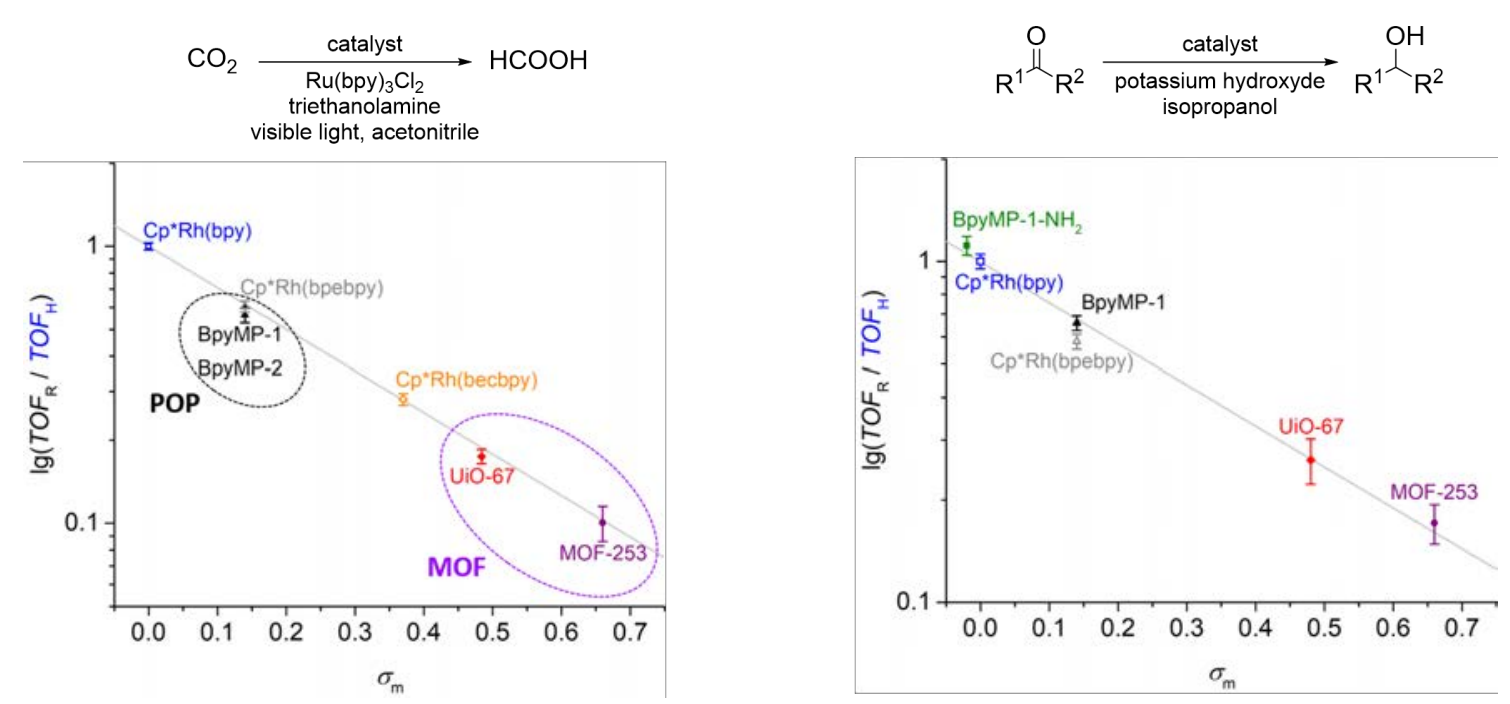

Figure 3. Linear correlation established for both homogeneous molecular and solid macroligand catalysts between calculated Hammett parameter ( $\left.\sigma_{\mathrm{m}}\right)$ and catalytic activity (TOF) for two reactions. (bpebpy: 5,5'-bis(phenyl-ethinylene)-2,2'-bipyridine, becbpy: bis-ethyl(2,2'-bipyridine)-5,5'-dicarboxylate). Adapted with permission from ${ }^{[7]}$ Copyright 2018 American Chemical Society and from ${ }^{[8]}$ Copyright 2018 Wiley-VCH Verlag GmbH \& Co. KGaA, Weinheim.

It has to be noted that the Hammett principle has been used previously to describe the influence of the MOF's linker substituents on the catalytic activity of the node itself. So far this principle has mainly been used for controlling the Lewis acidity of the metal-oxo node as originally reported by de Vos and coworkers for cyclization of citronellal, ${ }^{[33]}$ but has recently been extended towards electrocatalytic water oxidation by Tilley and co-workers. ${ }^{[34]}$ In our study, we used the Hammett parameter as a descriptor of the influence of the microporous macroligands on the catalytic activity of a heterogenized molecular catalyst.

In order to fully understand the behavior of the heterogeneous catalysts, their reactivity have been compared to those of their homogeneous counterparts and their activities plotted against their Hammett values $\left(\sigma_{\mathrm{m}}\right.$, Figure 3$)$. As a result, a linearity of $\sigma_{\mathrm{m}}$ against the respective turn-over frequency (TOF) was obtained. The authors deduced that local electronic effects drive the catalytic activity and that the reaction is therefore not diffusion limited.

Furthermore, the Hammett concept, which was originally developed for molecular organic reactions, underlines the singlesite and molecular nature of the active sites within these heterogeneous catalysts. Introducing amino groups with strong electron donating properties on the POP backbone (BpyMP-1$\mathrm{NH}_{2}$ ) in a precise matter (second meta-position with respect to the bipyridine binding sites), made it possible to obtain by-design a better performing catalyst for the ketone transfer hydrogenation as anticipated by the calculated Hammett value (Figure 3 ). ${ }^{[8]}$

\section{Increase stability of isolate atoms}

Recently, Lin and coworkers explained the stability of heterogenized $\mathrm{Pd}^{\prime \prime}$ atoms in $\mathrm{N}$-heterocyclic carbene-containing
POPs with the electron-donating or -withdrawing properties of the backbone. ${ }^{[30]}$ For more electron-donating macroligands they observed a lower Pd loading and a deactivation of the catalyst over several catalytic cycles of Suzuki-coupling, whereas more electron-withdrawing macroligands show no deactivation. They explained their observation with a stabilization of the Pd-NHC bond when a more electron-withdrawing macroligand was used.

\section{Conclusion}

In the view of porous macroligands, microporous solids possibly being MOFs, COFs or POPs behave like molecular ligands. The Hammett parameter, describing the change in electron density on the active site, is a valuable descriptor for their activities, as it is long-known from homogeneous catalysis. Thus, this parameter can serve (a) as a tool for rational design of new catalysts, their fine-tuning and adaptation to different reaction conditions, (b) to directly compare homogeneous and heterogeneous catalysts and (c) to evaluated whether or not the reaction inside the solid macroligand is hampered by diffusion or not. The selection of a microporous macroligand for a given reaction is guided by the known dependency of catalytic activity on electron density available from homogeneous catalysis. As simplified rule if the catalyst requires a low electron density MOFs should be considered as macroligand while POPs should be considered for catalyst requiring a high electron density. However also the stability of the macroligand (hydrolysis) or accessibility of the active site (pore size) have to be considered.

Macroligands allow controlling the electron density to stabilize molecular complexes and thus might represent new alternatives to overcome a pitfall in heterogeneous catalysis, ${ }^{[29]}$ achieving long-term stability at both molecular and material scale. The 
versatility of macroligands' structures is mostly limited by the capacity to synthesize monomers. New generations of porous solids will further unify the two worlds of homogeneous and heterogeneous catalysis.

\section{Acknowledgements}

F.M.W. gratefully acknowledges financial support from CNRS through Momentum 2018 excellence grant. The authors acknowledge funding from the European Union's Horizon 2020 research and innovation program through the $\mathrm{H}$-CCAT project under grant agreement No 720996.

Keywords: heterogenous catalysis • homogeneous catalysis • porous solids $\bullet$ macroligands $\bullet$ Hammett constant

[1] J. M. Basset, A. Choplin, J. Mol. Catal. 1983, 21, 95-108.

[2] C. Copéret, A. Comas-Vives, M. P. Conley, D. P. Estes, A. Fedorov, V. Mougel, H. Nagae, F. Núñez-Zarur, P. A. Zhizhko, Chem. Rev. 2016, 116, 323-421.

[3] P. Berruyer, M. Lelli, M. P. Conley, D. L. Silverio, C. M. Widdifield, G. Siddiqi, D. Gajan, A. Lesage, C. Copéret, L. Emsley, J. Am. Chem. Soc. 2016, 139, 849-855.

[4] M. K. Samantaray, E. Pump, A. Bendjeriou-Sedjerari, V. D'Elia, J. D. A. Pelletier, M. Guidotti, R. Psaro, J.-M. Basset, Chem. Soc. Rev. 2018, 47, 8403-8437.

[5] A. D. Pomogailo, D. Wöhrle, in Macromol. Complexes (Eds.: F. Ciardelli, E. Tsuchida, D. Wöhrle), Springer-Verlag Berlin Heidelberg, 1996, pages 11-130

[6] G. Gelbard, Comptes Rendus l'Académie des Sci. - Ser. IIC - Chem. 2000, 3, 757-764.

[7] F. M. Wisser, P. Berruyer, L. Cardenas, Y. Mohr, E. A. Quadrelli, A. Lesage, D. Farrusseng, J. Canivet, ACS Catal. 2018, 8, 1653-1661.

[8] F. M. Wisser, Y. Mohr, E. A. Quadrelli, D. Farrusseng, J. Canivet, ChemCatChem 2018, 10, 1778-1782.

[9] Z. Chen, E. Vorobyeva, S. Mitchell, E. Fako, M. A. Ortuño, N. López, S. M. Collins, P. A. Midgley, S. Richard, G. Vilé, J. Pérez-Ramírez, Nat. Nanotechnol. 2018, 13, 702-707.

[10] Z. Ren, Y. Liu, Y. Lyu, X. Song, C. Zheng, S. Feng, Z. Jiang, Y. Ding, J. Catal. 2019, 369, 249-256.

[11] P. Van Der Voort, D. Esquivel, E. De Canck, F. Goethals, I. Van Driessche, F. J. Romero-Salguero, Chem. Soc. Rev. 2013, 42, 39133955.
[12] G. Férey, Chem. Soc. Rev. 2008, 37, 191-214.

[13] D. Wu, F. Xu, B. Sun, R. Fu, H. He, K. Matyjaszewski, Chem. Rev. 2012 112, 3959-4015.

[14] J. Wu, F. Xu, S. Li, P. Ma, X. Zhang, Q. Liu, R. Fu, D. Wu, Adv. Mater 2018, 1802922.

[15] C. S. Diercks, O. M. Yaghi, Science 2017, 355, eaal1585.

[16] S. Kramer, N. R. Bennedsen, S. Kegnæs, ACS Catal. 2018, 6961-6982.

[17] V. Pascanu, G. González Miera, A. K. Inge, B. Martín-Matute, J. Am. Chem. Soc. 2019, 141, 7223-7234.

[18] S. Kousik, S. Velmathi, Chem. - A Eur. J. 2019, chem.201901987.

[19] W. Tu, Y. Xu, S. Yin, R. Xu, Adv. Mater. 2018, 30, 1707582.

[20] C. Kaes, A. Katz, M. W. Hosseini, Chem. Rev. 2000, 100, 3553-3590.

[21] W. R. Grüning, G. Siddiqi, O. V. Safonova, C. Copéret, Adv. Synth. Catal. 2014, 356, 673-679.

[22] X. Wang, I. Thiel, A. Fedorov, C. Copéret, V. Mougel, M. Fontecave, Chem. Sci. 2017, 8, 8204-8213.

[23] K. Matsui, Y. Maegawa, M. Waki, S. Inagaki, Y. Yamamoto, Catal. Sci. Technol. 2018, 8, 534-539.

[24] E. D. Bloch, D. Britt, C. Lee, C. J. Doonan, F. J. Uribe-Romo, H Furukawa, J. R. Long, O. M. Yaghi, J. Am. Chem. Soc. 2010, 132 14382-14384.

[25] M. I. Gonzalez, E. D. Bloch, J. A. Mason, S. J. Teat, J. R. Long, Inorg Chem. 2015, 54, 2995-3005.

[26] P. Kuhn, A. Thomas, M. Antonietti, Macromolecules 2009, 42, 319-326.

[27] D. B. Shinde, H. B. Aiyappa, M. Bhadra, B. P. Biswal, P. Wadge, S. Kandambeth, B. Garai, T. Kundu, S. Kurungot, R. Banerjee, J. Mater Chem. A 2016, 4, 2682-2690.

[28] Z. Dai, Q. Sun, X. Liu, L. Guo, J. Li, S. Pan, C. Bian, L. Wang, X. Hu, X. Meng, L. Zhao, F. Deng, F. S. Xiao, ChemSusChem 2017, 10, 11861192.

[29] U. I. Kramm, R. Marschall, M. Rose, ChemCatChem 2019, 11, 25632574.

[30] X. Liu, W. Xu, D. Xiang, Z. Zhang, D. Chen, Y. Hu, Y. Li, Y. Ouyang, H. Lin, New J. Chem. 2019, 43, 12206-12210.

[31] L. Wang, F. S. Xiao, ChemCatChem 2014, 6, 3048-3052.

[32] C. Hansch, A. Leo, R. W. Taft, Chem. Rev. 1991, 91, 165-195.

[33] F. Vermoortele, M. Vandichel, B. Van de Voorde, R. Ameloot, M Waroquier, V. Van Speybroeck, D. E. De Vos, Angew. Chem. Int. Ed. 2012, 51, 4887-4890.

[34] A. I. Nguyen, K. M. Van Allsburg, M. W. Terban, M. Bajdich, J. Oktawiec, J. Amtawong, M. S. Ziegler, J. P. Dombrowski, K. V. Lakshmi, W. S Drisdell, J. Yano, S. J. L. L. Billinge, T. D. Tilley, Proc. Natl. Acad. Sci. U. S. A. 2019, 116, 201815013 